\title{
MÉTODO PARA SIMULAÇÃO DE MOAGEM EM MOINHO DE BOLAS
}

\author{
W. L. GOMES ${ }^{1}$; H. DELBONI ${ }^{2}$; T. L. A. JATOBÁ ${ }^{3}$ e T. M. EL HAJJ ${ }^{4}$ \\ Universidade de São Paulo
}

wellingtonlacerdagomes@gmail.com ${ }^{1}$; hdelboni@usp.br²; tjatonline@gmail.com ${ }^{3}$; thammiris.poli@gmail.com ${ }^{4}$

Artigo submetido em novembro/2013 e aceito em dezembro/2013

DOI: http://dx.doi.org/10.15628/holos.2014.1782

\section{RESUMO}

O método de Bond para dimensionamento de moinho de bolas tem sido utilizado nos últimos 60 anos. Baseado no ensaio de WI este método é aplicável principalmente em circuitos tradicionais, os quais incluem estágios de britagem seguidos por moagem em moinhos de barras e bolas. Apesar da grande aplicação, este método apresenta algumas limitações. Por outro lado, a simulação é uma técnica reconhecida e aplicada para modelagem e simulação de circuitos de cominuição e classificação. Embora ambos os métodos sejam complementares, não há uma integração efetiva entre ambos. Este trabalho apresenta uma técnica que permite através da modelagem e simulação estimar e melhorar o desempenho de moagem em moinhos de bolas. O trabalho tem por base amostragem em circuitos industriais de moagem, bem como o desenvolvimento de ensaios de moagem em laboratório.

PALAVRAS-CHAVE: moagem, modelagem, simulação.

\section{METHOD FOR SIMULATING GRINDING IN BALL MILLS}

\begin{abstract}
The Bond method for ball mil designing has been used for 60 years. Based on Work Index test such method is applied mostly in conventional configuration, which includes multi-staged crushing followed by rod and ball milling. Despite the large application this method has some limitations. On the other hand simulation is a well spread and used technique for comminution and classification modeling and simulation. Even though both
\end{abstract}

methods are complementary there is no effective integration between them. This work shows a technique designed to estimate and improve the performance of grinding in ball mills through the modeling and simulation. The work is based on industrial data survey results, as well as on laboratory testing, the latter developed specifically for such a purpose

KEYWORDS: grinding, modelling, simulation 


\section{INTRODUÇÃO}

O principal método utilizado para dimensionamento de moinhos de bolas foi o proposto por Bond (1952). Este método, baseado na moabilidade determinada em laboratório, apesar da ampla aplicação em circuitos clássicos de cominuição (britagem estagiada, moagem em moinhos de barras seguida de moagem em moinhos de bolas) apresenta limitações em sua aplicação.

Devido às limitações do método de Bond para dimensionamento de circuitos industriais de moagem, a simulação tem sido cada vez mais utilizada para projetos de dimensionamento e de melhora de desempenho de circuitos de moagem em moinho de bolas. A grande vantagem da simulação em relação ao método proposto por Bond é a sua capacidade de avaliar o desempenho global de circuitos integrados de cominuição. Deste modo além de determinar a interação entre as diversas unidades do circuito, simulações permitem analisar e propor mudanças na configuração e tamanho dos equipamentos do circuito estudado. Além disso, a simulação fornece detalhes completos do circuito, incluindo distribuições granulométricas, vazões de sólidos e de água.

O modelo de balanço populacional (PBM) é o mais comumente utilizado (EPSTEIN, 1947) como base para modelos mecanísticos e fenomenológicos. Estes modelos, embora muito versáteis, apresentam pelo menos um grande problema, na medida em que os parâmetros não podem ser determinados a priori, mas devem ser ajustados aos dados de um circuito já existente. Utilizar dados de operação em planta piloto é uma opção, contudo isto requer uma quantidade de amostra de minério relativamente grande, a qual em estágios iniciais de desenvolvimento de empreendimentos mineiros pode não estar disponível. Além do mais, deve ser estabelecido um procedimento de scale-up que permita de maneira coerente e satisfatória o uso dos dados de operação em planta piloto. Testes em escala laboratorial resolvem o problema da quantidade de material da amostra, entretanto o procedimento de scale-up continua sendo um entrave ao método.

O presente trabalho teve como objetivo desenvolver um método que possibilite através da simulação estimar e melhorar o desempenho de moagem em moinho de bolas. $O$ desenvolvimento do método foi baseado em circuitos industriais de moagem com moinho de bolas, assim como, em ensaios laboratoriais.

\section{REVISÃO DA LITERATURA}

\subsection{Método de Bond}

O método empírico desenvolvido por Bond (1952) propõe caracterizar a resistência à moagem de minérios através do assim denominado Índice de Moabilidade ou Work Index (WI). $\mathrm{O}$ WI é uma característica do minério e indica o trabalho em kWh necessário para reduzir uma tonelada curta $(907 \mathrm{~kg}$ ) de material, desde um tamanho inicial infinito até um tamanho final de 80\% passante em $100 \mu \mathrm{m}$. Este índice é determinado laboratorialmente, através de um procedimento padronizado desenvolvido por Bond (1961). Embora característico do material, o valor do WI é função da relação de redução requerida para o circuito estudado. 
O método de Bond tem sido muito utilizado pela indústria mineral nas últimas décadas para dimensionamento de novas instalações de moagem. Isto se deve ao fato de que o método de Bond para caracterizar um minério pode ser ajustado para uma grande variedade de outros minérios.

Apesar da grande aplicação, o método de Bond é limitado em alguns aspectos como: (a) o método é aplicado para parâmetros e condições pré-estabelecidas por Bond, (b) o consumo energético é calculado em função do $\mathrm{P}_{80}$ e $\mathrm{F}_{80} \mathrm{e}$, portanto, não prevê a distribuição granulométrica completa do produto, (c) assume que a classificação dos circuitos é fixa e não permite analisar a interação entre as diferentes unidades do circuito e realizar mudanças na configuração e tamanho dos equipamentos.

Bond (1983) desenvolveu fatores de eficiência, os quais foram complementados por Rowland Jr. (1983), para correção da potência necessária aos moinhos e validade dos valores de WI determinados em laboratório, quando se estuda circuitos que diferem das condições préestabelecidas por Bond.

\subsection{Modelo do Misturador Perfeito}

O Modelo do Misturador Perfeito ou Perfect Mixing Model (PMM) proposto por Whiten (1976) considerado um caso particular do $P B M$, é um dos dois principais modelos empregados em modelagem de circuitos industriais de moagem.

O PMM baseia-se no equilíbrio de cada faixa granulométrica que compõe a carga do moinho, que está perfeitamente misturado. A carga do moinho está relacionada com o produto através de uma taxa de descarga para cada faixa granulométrica. Deste modo, as equações básicas do $P M M$ são definidas pelas equações 1 e 2 apresentadas a seguir.

$$
\begin{array}{ll}
f_{i}-p_{i}+\sum_{j=1}^{i-1} r_{j} s_{j} a_{i j}-r_{i} s_{i}\left(1-a_{i i}\right)=0 & \text { equação (1) } \\
p_{i}=d_{i} s_{i} \quad \text { equação (2) } &
\end{array}
$$

Onde:

$f_{i}=$ vazão de sólidos da alimentação do moinho, correspondente à i-ésima fração granulométrica $(\mathrm{t} / \mathrm{h})$;

$p_{i}=$ vazão de sólidos do produto do moinho, correspondente à i-ésima fração granulométrica $(\mathrm{t} / \mathrm{h})$;

$r_{j}=$ função taxa de quebra correspondente à j-ésima fração granulométrica $\left(\mathrm{h}^{-1}\right)$;

$s_{j}=$ massa da carga no interior do moinho, correspondente à j-ésima fração granulométrica $(\mathrm{t})$;

$a_{i j}=$ função distribuição de quebra, correspondente a fração da j-ésima faixa granulométrica,que aparece na i-ésima faixa granulométrica devido à fragmentação;

$d_{i}=$ taxa de descarga do moinho, correspondente à i-ésima fração granulométrica $\left(\mathrm{h}^{-1}\right)$. 
Segundo Napier-Munn et al. (1996) uma limitação de ordem prática importante, sob o ponto de vista da modelagem matemática, é a impossibilidade de se quantificar diretamente a distribuição granulométrica do minério contido na câmara interna dos moinhos. Para tanto, as duas equações básicas do $P M M$ foram associadas de forma a possibilitar a sua aplicação na modelagem de moinhos. Deste modo, chega-se à equação 3 que caracteriza o $P M M$.

$f_{i}-p_{i}\left(1-\frac{r_{i}}{d_{i}}\left(1-a_{i i}\right)\right)+\sum_{j=1}^{i-1} a_{i j}\left(\frac{r_{i}}{d_{i}}\right) p_{i}=0$

equação (3)

Portanto, o quociente $r / d$ torna-se o principal parâmetro do modelo, e pode ser determinado através da distribuição granulométrica da alimentação e do produto do moinho, além de uma função quebra que represente com fidelidade o minério processado (NAPIER-MUNN et al., 1996). A divisão do quociente $r / d$ pelo tempo de residência médio das partículas no interior do moinho leva à normalização do mesmo, independente das dimensões e condições operacionais do moinho estudado. O parâmetro $r / d^{*}$ normalizado é representado por uma curva quadrática definida por 3 ou no máximo 4 pontos. Para que se possa cobrir toda a faixa granulométrica estudada utiliza-se o método spline function.

\section{METODOLOGIA}

O trabalho consistiu na amostragem industrial do circuito de moagem da Mineração Serra Grande (MSG), com tomada de todos os fluxos como alimentação, produto e fluxos intermediários, assim como tomada dos dados físicos dos equipamentos e condições operacionais do circuito e, de uma amostra do produto da britagem primária.

Seguida da amostragem foi realizado o tratamento das amostras, o qual incluiu determinação da distribuição granulométrica, da densidade, da porcentagem de sólidos dos fluxos amostrados e a caracterização tecnológica do minério quanto à fragmentação, como Drop Weight Test (DWT), WI (executado na malha 0,212 mm - 65\# Tyler) e ensaios de moagem em escala de bancada. Os resultados desta etapa foram utilizados como base para a modelagem matemática do circuito industrial de moagem amostrado e definição dos parâmetros para simulação do mesmo.

Os ensaios de moagem em laboratório foram realizados em moinho de diâmetro e comprimento iguais a 10" (254 mm), a 70\% de sólidos e 33\% de grau de enchimento. As condições operacionais variadas para cada ensaio estão apresentadas na Tabela 1 a seguir.

Tabela 1 - Condições Operacionais dos Ensaios de Moagem em Laboratório.

\begin{tabular}{c|c|c|c}
\hline ENSAIOS & MOINHO & $\begin{array}{c}\text { \% VELOCIDADE } \\
\text { CRÍTICA }\end{array}$ & $\begin{array}{c}\text { TAMANHO MÁXIMO } \\
\text { DE BOLA (mm) }\end{array}$ \\
\hline Ensaio 1 & Com Aletas & 72 & 38 \\
\hline Ensaio 2 & Com Aletas & 75 & 38 \\
\hline Ensaio 3 & Com Aletas & 69 & 38 \\
\hline Ensaio 4 & Com Aletas & 72 & 30 \\
\hline Ensaio 5 & Com Aletas & 72 & 25 \\
\hline Ensaio 6 & Sem Aletas & 72 & 38 \\
\hline
\end{tabular}




\subsection{Método}

O método utilizado no presente tem como base os trabalhos desenvolvidos por Morrell e Man (1997) e Man (2001a, b, c). O método necessitou de quatro componentes principais, os quais são listados a seguir.

Ensaios de moagem e caracterização tecnológica em laboratório: tiveram como objetivo fornecer os parâmetros para calibração dos modelos do circuito de moagem estudado, como appearance function, breakage rate e vazão de sólidos;

Modelo matemático para moinho de bolas: o PMM foi utilizado para calibrar o circuito de moagem, a partir dos parâmetros obtidos pelos ensaios de laboratório, bem como prever o seu desempenho para diferentes condições através dos trabalhos de simulação.

Critérios para scale-up: definidos a partir da comparação dos resultados dos ensaios de moagem em laboratório, com os dados obtidos a partir da amostragem do circuito industrial;

Programa computacional de simulação: o software para simulação além de ser uma ferramenta matemática poderosa, teve como função integrar os modelos de moagem e classificação, e simular o desempenho global do circuito de moagem. Para estes fins foi utilizado o software JKSimMet.

\section{RESULTADOS}

Foram realizadas modelagens para cada ensaio de laboratório assim como para o circuito industrial da MSG, a partir das distribuições granulométricas e condições operacionais.

A partir dos parâmetros de moagem calibrados para cada ensaio de moagem em laboratório foi realizada a simulação para prever o produto do circuito industrial (overflow dos hidrociclones).

A simulação consistiu em estimar o produto do circuito industrial a partir dos parâmetros calibrados de moagem em laboratório, da alimentação nova dos circuitos industriais e do tamanho de bolas industriais, parâmetro o qual descreve como a potência aplicada ao moinho é transferida à carga no interior do moinho (MORRELL; MAN, 1997).

O gráfico da Figura 1 apresenta os resultados comparativos entre as distribuições granulométricas estimadas através das simulações com a do produto do circuito industrial, e também com aquela estimada pelo ensaio de WI de Bond. 


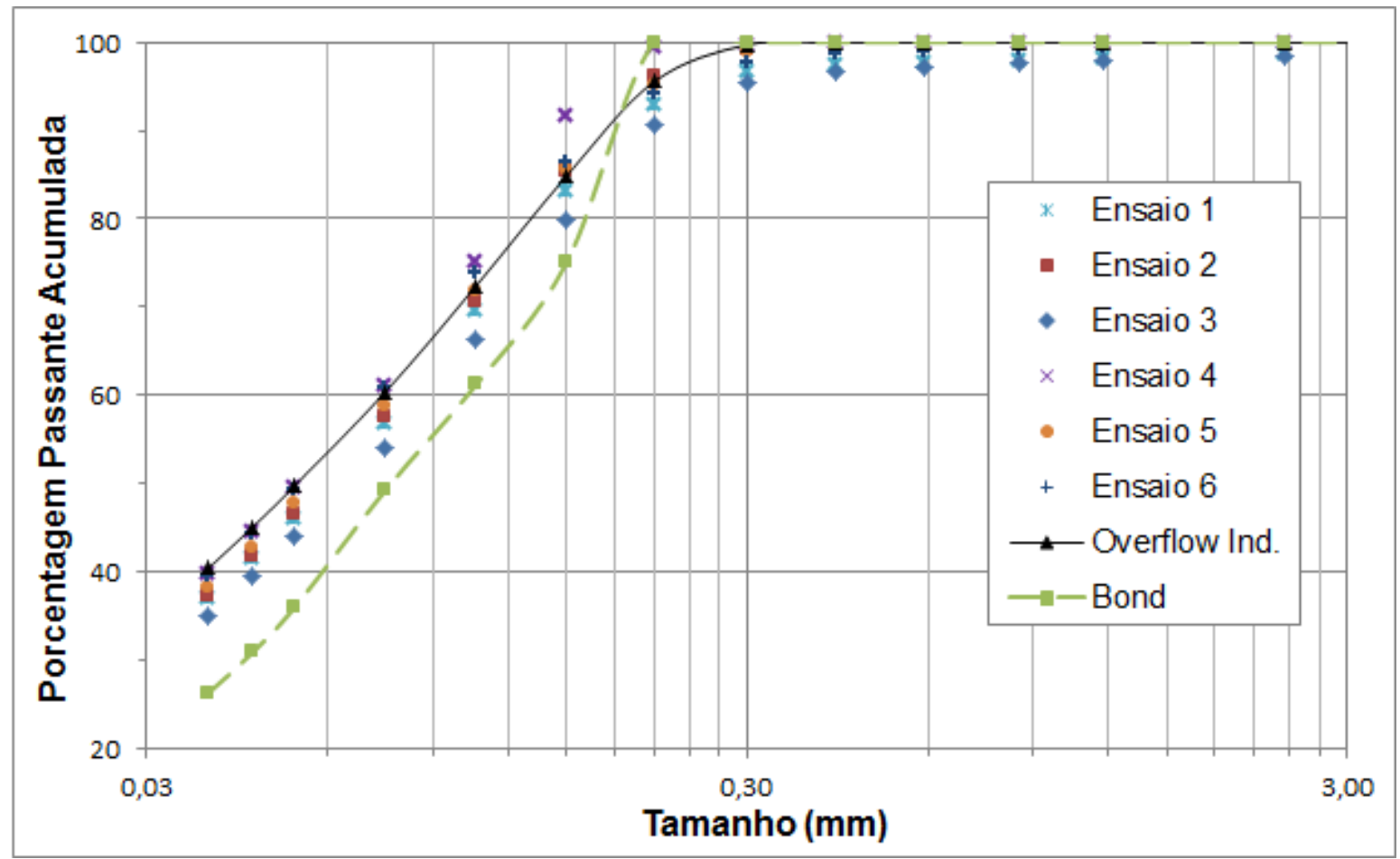

Figura 1 - Comparação entre as Distribuições Granulométricas Estimadas em Laboratório e a Industrial.

Pelo gráfico da Figura 1, observa-se que as curvas de distribuição granulométrica simuladas são bastante próximas à curva do produto industrial e, também possuem formatos similares. Já a curva estimada pelo método de Bond apresentou distribuição e formato bastante diferentes.

A Tabela 2 apresenta os valores de $\mathrm{P}_{80}$ estimados pelos ensaios de laboratório e o erro relativo em relação ao $\mathrm{P}_{80}$ industrial.

Tabela 2 - Resultados de $\mathbf{P}_{80}$ estimados e industrial.

\begin{tabular}{c|c|c}
\hline ENSAIOS & $\mathbf{P}_{\mathbf{8 0}}(\mathbf{m m})$ & ERRO $(\%)$ \\
\hline Ensaio 1 & 0,137 & 5,4 \\
\hline Ensaio 2 & 0,131 & 0,8 \\
\hline Ensaio 3 & 0,151 & 16,2 \\
\hline Ensaio 4 & 0,116 & 10,8 \\
\hline Ensaio 5 & 0,129 & 0,8 \\
\hline Ensaio 6 & 0,125 & 3,8 \\
\hline Bond & 0,160 & 23,1 \\
\hline Industrial & 0,130 & - \\
\hline
\end{tabular}

Em relação ao parâmetro $\mathrm{P}_{80}$, os ensaios 2 e 5 apresentaram a melhor estimativa em relação ao industrial com erro de $0,8 \%$. O método de Bond apresentou o resultado mais distante com erro de $23,1 \%$.

A Tabela 3 apresenta a matriz de proximidade com índices de dissimilaridade comparando os ensaios de moagem em laboratório e o ensaio de WI em relação ao circuito industrial. Este 
índice representa a raiz quadrada da soma dos quadrados das diferenças entre porcentagem passante simulada e industrial

Tabela 3 - Matriz de proximidade com índices de dissimilaridade.

\begin{tabular}{c|c|c|c|c|c|c|c|c}
\hline ENSAIOS & $\mathbf{1}$ & $\mathbf{2}$ & $\mathbf{3}$ & $\mathbf{4}$ & $\mathbf{5}$ & $\mathbf{6}$ & BOND & INDUSTRIAL \\
\hline $\mathbf{1}$ & 0 & 6,278 & 7,110 & 14,706 & 7,073 & 8,765 & 24,575 & 10,003 \\
\hline $\mathbf{2}$ & 6,278 & 0 & 12,166 & 10,270 & 2,601 & 6,917 & 25,103 & 6,573 \\
\hline $\mathbf{3}$ & 7,110 & 12,166 & 0 & 21,615 & 13,535 & 15,696 & 20,556 & 16,491 \\
\hline $\mathbf{4}$ & 14,706 & 10,270 & 21,615 & 0 & 8,989 & 8,110 & 33,913 & 8,424 \\
\hline $\mathbf{5}$ & 7,073 & 2,601 & 13,535 & 8,989 & 0 & 4,628 & 27,449 & 4,392 \\
\hline $\mathbf{6}$ & 8,765 & 6,917 & 15,696 & 8,110 & 4,628 & 0 & 31,471 & 3,985 \\
\hline BOND & 24,575 & 25,103 & 20,556 & 33,913 & 27,449 & 31,471 & 0 & 30,890 \\
\hline INDUSTRIAL & 10,003 & 6,573 & 16,491 & 8,424 & 4,392 & 3,985 & 30,890 & 0 \\
\hline
\end{tabular}

Analisando a matriz de proximidade da Tabela 3, nota-se que a maior dissimilaridade ocorre para a estimativa pelo método de Bond $(30,890)$. Portanto, todas as estimativas da distribuição granulométrica industrial através da modelagem e simulação apresentaram resultados mais satisfatórios do que o método de Bond. O ensaio 6 apresentou o melhor resultado com distância euclidiana de 3,985. A Figura 2 apresenta o gráfico comparativo entre porcentagem passante industrial e simulada pelo ensaio 6.

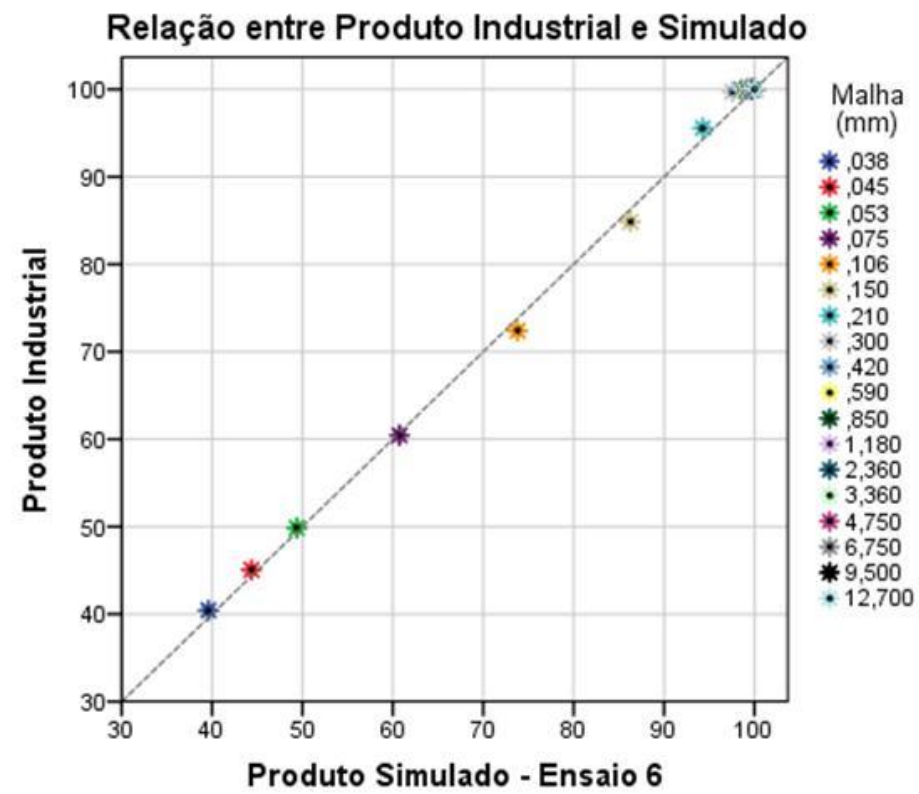

Figura 2 - Gráfico produto industrial versus produto simulado - ensaio 6.

As porcentagens passantes do produto simulado do ensaio 6 foram aproximadas por uma reta através de regressão linear, apresentando um alto coeficiente de determinação $\left(r^{2}=0,999\right)$, sugerindo que a relação entre as porcentagens passantes industriais e simuladas é linear. Ainda, observou-se uma alta aderência entre as porcentagens passantes industriais e simuladas, indicando uma relação próxima de 1:1, representada pela bissetriz traçada na Figura 2. A regressão 
linear do produto industrial versus produto simulado do ensaio 6 apresentou coeficientes linear e angular de 0,023 e 1,004, respectivamente, comprovando que a estimativa gerada pelo simulação foi aderente aos resultados industriais.

\section{CONCLUSÕES}

O método para estimar e melhorar o desempenho de moagem em moinho de bolas através da modelagem e simulação apresentou bons resultados. Em relação à estimativa da distribuição granulométrica do produto industrial os resultados foram todos superiores ao método de Bond.

Portanto, o método apresentado para modelagem e simulação de moagem em moinho de bolas através de ensaios de laboratório é promissor. Entretanto, recomenda-se que o método seja aplicado a diferentes circuitos industriais de moagem em moinho de bolas, a fim de obter-se uma ampla validação do mesmo. Deste modo, o método poderá ser aplicado para integração completa de circuitos de moagem em moinho de bolas, possibilitando simular melhorias de processo.

\section{REFERÊNCIAS BIBLIOGRÁFICAS}

1. BOND, F.C. The Third Theory of Comminution. Transactions AIME, p 484-494, May, 1952.

2. BOND, F.C. Crushing and Grinding Calculations. Allis Chalmers Publication,1961.

3. BOND, F.C. Crushing and Grinding Calculations. Selection Circuits to Prepare Beneficiation Feeds. Allis Chalmers Bulletin, May, 1983.

4. EPSTEIN, B. The Material Description of Certain Breakage Mechanisms Leading to the Logorithmic-Normal Distribution. J. Franklin Inst, 1947.

5. MAN, Y. T. Model-Based procedure for scale-up of wet, overflow ball mills. Part I. Outline of the methodology. Minerals Engineering, v. 14, n. 10, p. 1237-1246, Oxford, 2001a.

6. MAN, Y.T. Model-Based procedure for scale-up of wet, overflow ball mills. Part II. Worked example. Minerals Engineering, Oxford, v. 14, n. 10, p. 1247-1257, 2001b.

7. MAN, Y.T. Model-Based procedure for scale-up of wet, overflow ball mills. Part III. Validation and discussion. Minerals Engineering, Oxford, v. 14, n. 10, p. 1259-1265, 2001c.

8. MORRELL, S., MAN, Y.T. Using modelling and simulation for the design of full scale ball mill circuits. Minerals Engineering, Oxford, v. 10, n. 12, p. 1311-1327, 1997.

9. NAPIER-MUNN, T.J., MORRELL, S., MORRISON, R.D., KOJOVIC, T. Mineral Comminution Circuits: their operation and optimization. Queensland (JKMRC), 413 p, 1996.

10. ROWLAND JR, C.A. Selection of Rod Mills, Ball Mills, Pebble Mills and Regrind Mills. Selection Circuits to Prepare Beneficiation Feeds. Allis Chalmers Bulletin, May, 1983.

11. WHITEN, W.J. Ball mill simulation using small calculators. Proceedings AusIMM, p. 47-53, 1976. 